\title{
Monitoring Selfish Nodes in MANET during Replica Allocation
}

\author{
E. Jananandhini ${ }^{1}$ \\ ${ }^{I}$ (Department of Computer Science and Engineering, P. A. College of Engineering and Technology, India)
}

\begin{abstract}
Mobile Ad Hoc Network is a collection of mobile hosts. The mobility nature of the MANET causes the mobile host to move freely in the network, due to that network disconnections occurs frequently and this causes frequent changes in the network topology. Data in one network cannot be accessed by the host in the another network, the data accessibility from one network to another network becomes impossible, consequently data accessibility become lower in the wireless network than in the conventional fixed network. This leads to performance degradation. Many Replica allocation methods are used to improve the data accessibility by replicating data item on mobile hosts other than the original owner of the data. This improves the data accessibility but some of the mobile hosts in the network may act as selfish nodes. These nodes use the services of other nodes but this does not cooperate with other nodes, this reduces the overall data accessibility. Selfish node detection algorithm is used to detect the selfish nodes and then the data replica allocation method is used for data replication. The simulation result shows that the conducted selfish node detection method and replica allocation techniques outperforms the improvement in data accessibility and reduce the communication cost and query delay.
\end{abstract}

Keywords: Data accessibility, Selfish Nodes, Degree of selfishness, Mobile adhoc network, Replica allocation.

\section{Introduction}

Mobile Ad Hoc Network (MANET) is a collection of mobile nodes that communicate over relatively low capacity wireless links, without a centralized infrastructure nodes can communicate with each other, there will be no access points for communication. Each node in the MANET acts as a router and communicate with $[10,11]$ each other. In these networks, the mobility nature of the node and the wireless communication links may lead to dynamically changing and highly volatile topologies. All network functions routing, multi-hop packet delivery and mobility management have to be performed by the member nodes itself, individually or collectively [7]. The network performance becomes highly dependent on collaboration of all member nodes.

\section{A. Data Replication Technique}

In Manet, the mobile hosts transit freely due to this disconnections occurs many time and causes frequent network division. Consequently, different fundamental technologies [5] from those of conventional fixed networks are needed. One whole network is divided into two or more networks due to the migrations of mobile hosts, [8] mobile hosts in one of the divided two networks cannot access data items held by mobile hosts in the other network. The data accessibility in ad hoc networks is lower than that in conventional fixed networks. In ad hoc networks, preventing deterioration of data accessibility at the point of network division is a very important issue. A solution is to replicate the data items on mobile hosts other than the original owners.

\section{B. Selfish Nodes}

The presence of selfish nodes is a major issue in mobile ad-hoc network [16], it reduces the data accessibility. Data accessibility mainly involves cooperation of all nodes in the network but the presence of selfish nodes causes the data accessibility. A non-cooperative action of misbehavior is usually termed as selfishness, notably different from malevolent behavior. Selfish nodes utilize the network in favor of its own communication, [3] but simply refuse to cooperate in forwarding packets for other nodes in order to save battery consumption. Selfish nodes thus utilize the reimbursement provided by the resources of other nodes, but will not offered its own resources to help others. The behavioral states for nodes from the viewpoint of selfish replica allocation are of three types [14].

1) Type-1 node: The nodes are non selfish nodes. The nodes hold replicas allocated by other nodes within the limits of their memory space.

2) Type-2 node: The nodes are partially selfish nodes. The nodes sometimes allow the other nodes to share their memory for allocation.

3) Type-3 node: The nodes are fully selfish nodes. This does not shares its memory with other nodes, but uses others nodes memory for their own purpose. 


\section{Related Works}

There are several techniques used for detection of selfish node and several data replica allocation methods are used for improving the data accessibility. The acknowledgement based scheme [1] as the TWOACK and S-TWOACK improves the end-to-end packet delivery ratio, this can be added on to the source routing protocol such as DSR protocol, this causes the routing overhead. Time based detection schemes are used based upon the response time of the packets [13], but this failed to detect the neighboring nodes. In multi Hop ack scheme [15] N-ACK and CNAP schemes are used. Some techniques use the watchdog, pathrater, random feedback, distributed reputation, Mobile IDS and currency systems. These cause the overall network overhead. Some sensors [9] are used for detection of such malicious nodes such as Activity-Based Overhearing, Iterative probing, Unambiguous-Probing, if multiple sensors are active in parallel and a selfish node is detected by a number of these sensors. But using sensor in detecting the selfish node becomes a tedious process. The novel algorithm [4] is used but it is failed to detect some topologies. In the reputation based scheme [12], the watchdog and pathrater models are used, in the credit based scheme the packet purse model and packet trade model is used, the basic idea in this scheme is to provide incentives for nodes to faithfully perform networking functions. The data replica allocation [2] models such as Static Access Frequency, only the data frequency to each data item is taken out. In Dynamic Access Frequency and Neighborhood, the access frequency to each item and neighborhood among mobile hosts is taken and in the Dynamic Connectivity based grouping, the whole network topology is taken into account. In all these models the periodic updating of data item is not considered [15].

\section{Proposed Work}

In this paper, the formula is used to detect the selfish nodes in the network. The data replica allocation technique is used to improve the data accessibility.

\section{Detection of Selfish Nodes}

The Credit Risk (CR) value is calculated for each node in the network shown in the equation (1), each node calculates the CR score to each of its connected node [17].

$$
\text { Acclaim risk }=\frac{\text { Estimated risk }}{\text { Estimated value }}
$$

The node estimates the degree of selfishness for all of its connected nodes based on the score. The selfish feature is described first, that may lead to the selfish replica allocation problem to determine both expected value and expected risk.

The two categories of selfish features are node-specific and query processing-specific. Node-specific features can be explained by considering the following case: A selfish node may share part of its own memory space like type-3 node [6]. The size of shared memory space or the number of shared data items can be used to represent the degree of selfishness, the size of $\mathrm{N}_{\mathrm{k}}$ 's shared memory space, denoted as $\mathrm{SS}_{\mathrm{i}}^{\mathrm{k}}$, and the number of $\mathrm{N}_{\mathrm{k}}$ 's shared data items, denoted as $\mathrm{ND}^{\mathrm{k}}{ }_{\mathrm{i}}$, observed by a node $\mathrm{N}_{\mathrm{i}}$.

The query processing-specific feature is the ratio of selfishness alarm of $\mathrm{N}_{\mathrm{k}}$ on Ni, denoted as $\mathrm{P}^{\mathrm{k}}{ }_{\mathrm{i}}$, is the ratio of $\mathrm{N}_{\mathrm{i}}$ 's data request being not served by the expected node $\mathrm{N}_{\mathrm{k}}$ due to $\mathrm{N}_{\mathrm{k}}$ 's selfishness in its memory space. The query processing-specific feature can represent the expected risk of a node. The $\mathrm{P}_{\mathrm{i}}^{\mathrm{k}}$ gets larger, node $\mathrm{N}_{\mathrm{i}}$ will treat $\mathrm{N}_{\mathrm{k}}$ as a risky node because a large $\mathrm{P}^{\mathrm{k}}$ i means that $\mathrm{N}_{\mathrm{k}}$ cannot serve $\mathrm{N}_{\mathrm{i}}$ 's requests due to selfishness in its memory usage. $\mathrm{N}_{\mathrm{i}}$ should know the status of other nodes' memory space to effectively identify the expected nodes memory space.

$$
\mathrm{CR}_{\mathrm{i}}^{\mathrm{k}}=\frac{\mathrm{P}_{\mathrm{i}}^{\mathrm{k}}}{\alpha \times \mathrm{SS}_{\mathrm{i}}^{\mathrm{k}}+(1-\alpha) \times \mathrm{ND}_{\mathrm{i}}^{\mathrm{k}}}
$$

The system parameter, $\alpha$, is used to adjust the relative importance of $\mathrm{SS}^{\mathrm{k}}{ }_{\mathrm{i}}$ and $\mathrm{ND}_{\mathrm{i}}^{\mathrm{k}}$. Node $\mathrm{N}_{\mathrm{i}}$ updates $\mathrm{CR}_{\mathrm{i}}^{\mathrm{k}}$ at every query processing and looks it up for the connected node $\mathrm{N}_{\mathrm{k}}$ at every relocation period, each node has its own threshold $\alpha$, of $\mathrm{CR}_{\mathrm{i}}^{\mathrm{k}}$. The measured $\mathrm{CR}_{\mathrm{i}}^{\mathrm{k}}$ shown in the equation (2), exceeds $\alpha$ node $\mathrm{N}_{\mathrm{k}}$ will be detected as a selfish node by $\mathrm{Ni}$. The value of $\mathrm{P}^{\mathrm{k}}{ }_{\mathrm{i}}$ is updated at every query processing of some item that $\mathrm{Ni}$ allocates to other node or nodes during the replica allocation phase. The effect of parameters $\mathrm{SS}_{\mathrm{i}}^{\mathrm{k}}$ and $\mathrm{ND}_{\mathrm{i}}^{\mathrm{k}}$ on $\mathrm{CR}^{\mathrm{k}}{ }_{\mathrm{i}}$ can be weighted by taking into consideration the size of memory space at node $\mathrm{Ni}$, $\mathrm{Si}$, and the total number of data items accessed by $\mathrm{Ni}$. 


\section{Replica Allocation methods}

The Three proposed new replica allocation methods are the extensions of the previous replica allocation methods [2] and to reduce the network traffic and periodic update of data in the mobile hosts. The three extended methods are as follows:

1. Extended Static Access Frequency (E-SAF) method

2. Extended Dynamic Access Frequency Neighborhood (E-DAFN) method

3. Extended Dynamic Connectivity based Grouping (E-DCG) method.

The PT value can be calculated as shown in the equation (3):

$$
\mathrm{p}_{\mathrm{ij}} \cdot \tau_{\mathrm{j}}=\mathrm{p}_{\mathrm{ij}} \cdot\left(\mathrm{T}_{\mathrm{j}}-\mathrm{t}_{\mathrm{j}}\right)
$$

$\mathrm{p}_{\mathrm{ij}}$ is the probability of an access request for data item $\mathrm{Dj}$ from mobile host Mi is issued at a unit of time, that is the access frequency, $\tau \mathrm{j}$ is the time remaining until $\mathrm{Dj}$ is updated next, $\mathrm{Tj}$ denotes the update period of $\mathrm{Dj}$, t $\mathrm{j}$ denotes the time that has passed, $\mathrm{Dj}$ has been updated at the most recent update period. The PT value represents the average number of access requests that are issued for $\mathrm{Dj}$ until it is updated next, and it takes the maximum value $\mathrm{p}_{\mathrm{ij}}$. By allocating replicas with high PT values at a relocation period, the data accessibility is expected to be higher. These three methods are proposed by mainly changing their algorithms to use PT values instead of access frequencies [16].

2.1 E-SAF method: The memory space for the replica is temporarily filled with one of the replicas that have been allocated the previous relocation period but are not currently selected for allocation.[16] This temporarily allocated replica is chosen from among the possible replicas according to that have the highest PT value. The memory space remains free, if there is no replica that can be temporarily allocated. The data access to the data item the replica should be allocated succeeds, the memory space is filled with the valid replica.

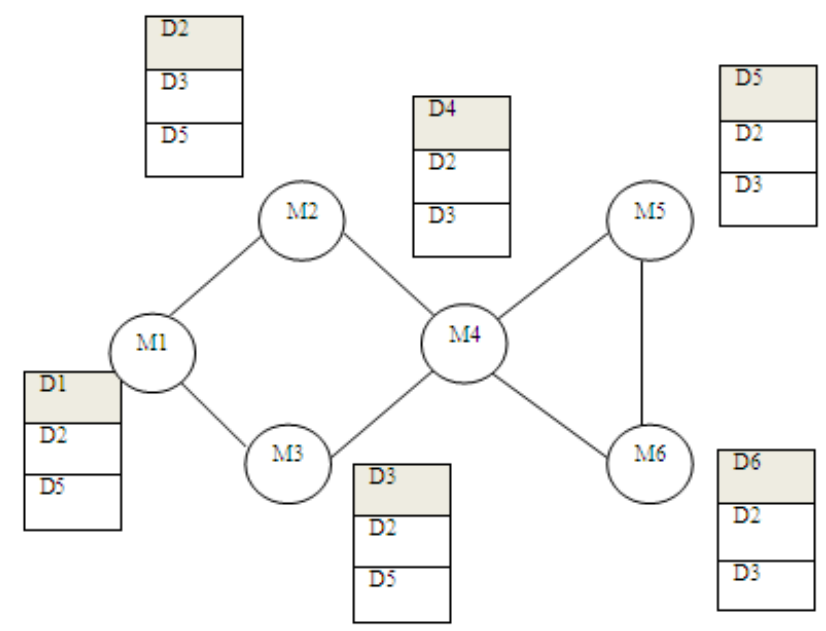

Fig.1 An example for E-SAF method

The Fig 1 shows the example diagram of E-SAF method. The mobile hosts do not need to exchange information with each other for replica allocation in the E-SAF method, replica allocation can be done with low overhead and low traffic. On the other hand, the each mobile host allocates replicas based on the PT values to the data items, mobile hosts with the same access characteristics allocate the same replicas. A mobile host can access data items or replicas held by other connected mobile hosts, it is more effective to share many kinds of replicas among them.

2.2 E-DAFN method: The E-DAFN solves the problem with the E-SAF method, this method eliminates replica duplication among neighboring mobile hosts.[16] There exists a replica duplication of a data item between two neighboring mobile hosts, the mobile host with lower PT value to the data item replaces the replica with another replica. The Fig 2 shows the example diagram of E-DAFN method. 


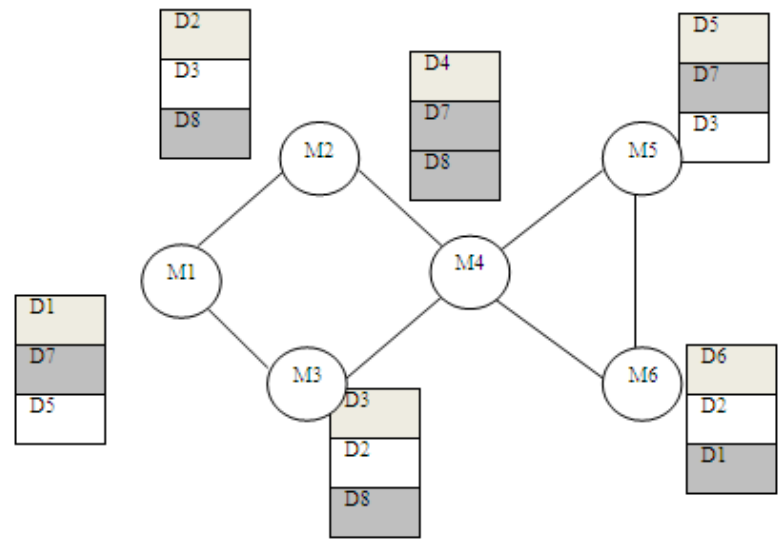

Fig. 2 An example for E-DAFN method

The algorithm of E-DAFN method is,

Step 1: At a relocation period, each mobile host broadcast its host identifier and information on access frequencies to data items. After all mobile hosts complete their broadcasts, from the received host identifiers, every host knows its connected mobile hosts.

Step 2: Each mobile host determines the preliminary allocation of replicas based on the E-SAF method.

Step 3: In each set of mobile hosts that are connected to each other, starting from the mobile host with the lowest suffix i of host identifier Mi, the following procedure is repeated in the order of the breadth first search. There exists a duplication of a data item between two neighboring mobile hosts, and if one of them is the original, the host that holds the replica replaces it with another replica. The host having PT value to the data item is lower replaces the replica with another replica, if both of them are replicas.

At a relocation period, a mobile host might not connect to another mobile host that has an original or a replica of a data item that the host should allocate. In E-SAF method, the memory space for the replica is temporarily filled with another replica, and is later filled with the valid replica during data access to the data item succeeds.

\subsection{E-DCG method:}

The E-DCG method shares replicas in each larger group of mobile hosts than the E-DAFN method that shares replicas among neighboring hosts. [16] In order to share replicas effectively, each group should be stable, i.e., not easily divided due to changes in network topology. The E-DCG method creates groups of mobile hosts that are biconnected components in a network. A biconnected component denotes a maximum partial graph is connected if an arbitrary node in the graph is deleted. By grouping mobile hosts as biconnected components, the group is not divided even if one mobile host dis appears from the network or one link is disconnected in the group and it is considered that the group has high stability. The Fig.3 is the example diagram of E-DCG method.

The algorithm of E-DCG method is,

Step 1: At a relocation period, each mobile host broadcast sits host identifier and information on access frequencies to data items. After all mobile hosts complete their broadcasts, every host knows its connected mobile hosts.

Step 2: In each set of mobile hosts that are connected to each other, starting from the mobile host with the lowest suffix of host identifier (Mi), an algorithm to find biconnected components is executed. Each biconnected component is put in a group.

Step 3: In each group, a group access frequency to each data item is calculated as a summation of access frequencies of mobile hosts in the group to the item. The PT value of the group to each item is calculated. These calculations are done by the mobile host with the lowest suffix of host identifier in the group.

Step 4: In descending order of PT values in each group, replicas are allocated until the memory space of all mobile hosts in the group becomes full. The replicas of data items that are held as originals by mobile hosts in the group are not allocated. Each replica is allocated at a mobile host having the PT value to the data item is the highest among hosts that have free memory space to create it.

Step 5: After allocating replicas of all data items that have no original in the group, the free memory space still exists, each replica is allocated at a mobile host the PT value to the data item is the highest among hosts that have free memory space to create it and do not hold a replica or the original of the item. The replica is not allocated if there is no such mobile host. 


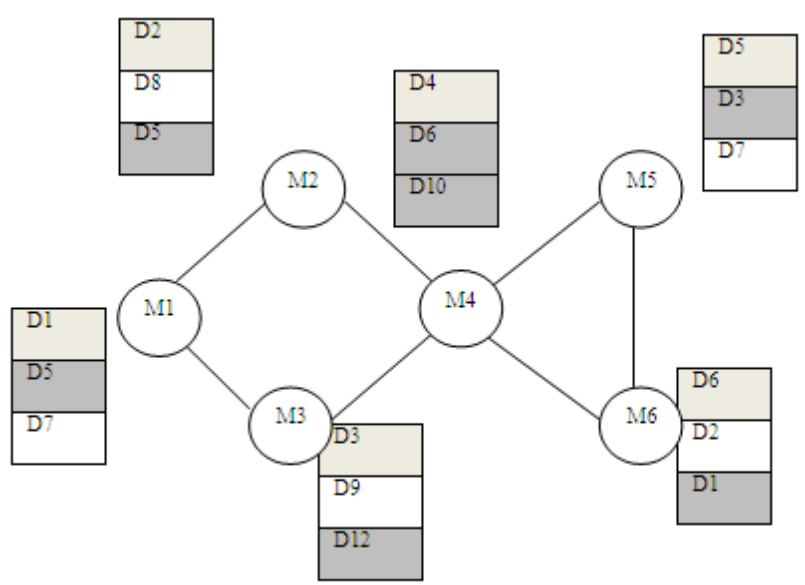

Fig.3 An example for E-DCG method

\section{Results And Discussion}

The proposed technique is done using Network Simulator, this technique improves the data accessibility by detecting the selfish nodes, the selfish node detection is done using formulae.

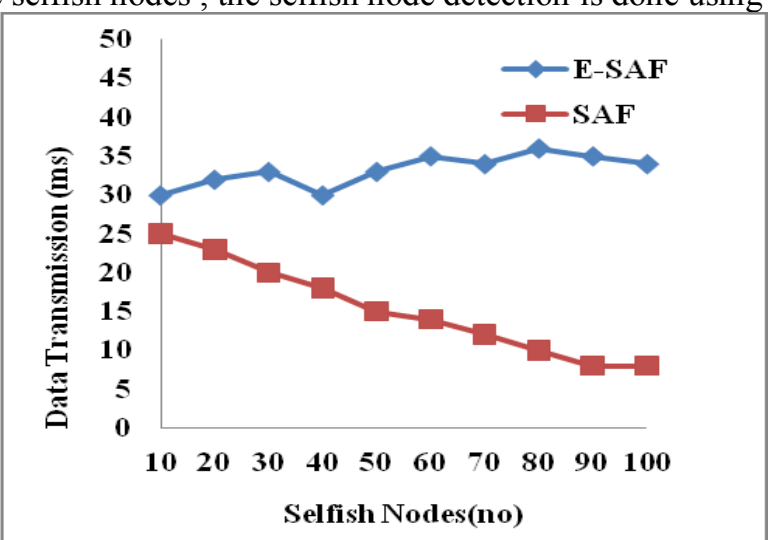

Fig.4 Comparison of E-SAF and SAF method

The data replica allocation technique is used to improve the data transmission between the nodes. The Fig. 4 shows the data transmission comparision between the SAF and E-SAF method. In the presence of selfish nodes.in E-SAF method the data trasmission is constant but in the SAF method it become low. Next the Fig. 5 shows the data transmission comparision between the E-DAFN and DAFN method. The E-DAFN method transmits at constant rate than the DAFN method.

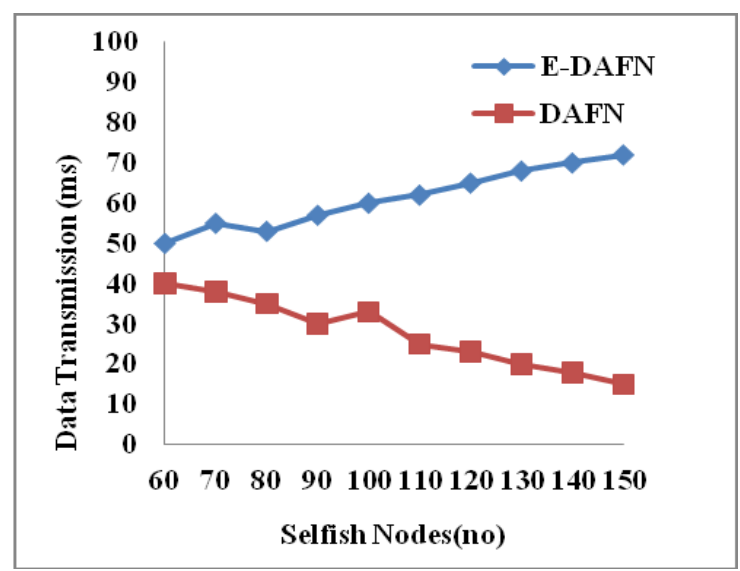

Fig.5 Comparison of E-DAFN and DAFN method 


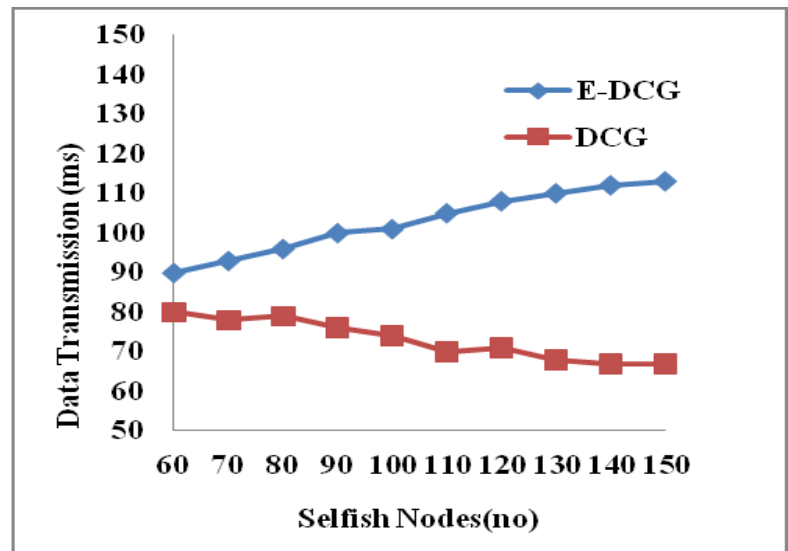

Fig.6 Comparison of E-DCG and DCG method

The Fig 6 shows the comparision between E-DCG and DCG methods. Comparing to previous E-SAF and E-DAFN method, the data transmission is higher in the E-DCG method. Among the three methods the EDCG method transmits data at high rate in the presence of selfish nodes.

\section{Conclusion}

In the mobile ad hoc network, each data item is periodically updated and the proposed three replica allocation methods are used to improve the data accessibility. In the E-SAF method, a mobile host allocates replicas with high PT values. In the E-DAFN method, from replica allocation based on the E-SAF method, replica duplication is eliminated among neighboring mobile hosts. In the E-DCG method, replicas are shared in each stable group of mobile hosts. In the proposed three methods, the E-DCG method gives the highest accessibility and the E-SAF method gives the lowest traffic. The mobile hosts are connected by unstable radio links that are likely to be disconnected after a short time, it is inefficient to allocate different replicas on them because the mobile hosts cannot share the replicas after disconnections, and it is suitable for only stable link mobile hosts, the further extension is to allocate the replicas in unstable links.

\section{References}

[1] K. Balakrishnan, J. Deng, and P.K. Varshney, "TWOACK: Preventing Selfishness in Mobile Ad Hoc Networks", Proc. IEEE Wireless Comm. and Networking, pp. 2137-2142, 2005.

[2] T. Hara, "Effective Replica Allocation in Ad Hoc Networks for Improving Data Accessibility", Proc. IEEE INFOCOM, pp. 1568$1576,2001$.

[3] S. Marti, T. Giuli, K. Lai, and M. Baker, "Mitigating Routing Misbehavior in Mobile Ad hoc Networks", Proc. ACM MobiCom. $255-265,2000$.

[4] H. Miranda and L. Rodrigues, "Friends and Foes: Preventing Selfishness in Open Mobile Ad hoc Networks", Proc. IEEE Int'l Conf. Distributed Computing Systems Workshops, pp. 440-445, 2003.

[5] P. Padmanabhan, L. Gruenwald, A. Vallur, and M. Atiquzzaman, "A Survey of Data Replication Techniques for Mobile Ad Hoc Network Databases”, The Int'1 J. Very Large Data Bases, vol. 17, no. 5, pp. 1143-1164, 2008.

[6] K. Paul and D. Westhoff, "Context Aware Detection of Selfish Nodes in DSR Based Ad-Hoc Networks", Proc. IEEE Global Telecomm. Conf., pp. 178-182, 2002.

[7] W. Wang, X.-Y. Li, and Y. Wang, "Truthful Multicast Routing in Selfish Wireless Networks”, Proc. ACM MobiCom, pp. 245-259, 2004.

[8] L. Yin and G. Cao, "Balancing the Tradeoffs between Data Accessibility and Query Delay in Ad Hoc Networks", Proc. IEEE Int'l Symp. Reliable Distributed Systems, pp. 289-298, 2004.

[9] Frank Kargl, Andreas Klenk, Michael Weber and Stefan Schlott, "Sensors for Detection of Misbehaving Nodes in MANETs", Department of Multimedia Computing University of Ulm, 2009.

[10] Takahiro Hara, "Replica Allocation Methods in Ad Hoc Networks with Data Update", Mobile Networks and Applications 8, 343354, 2003.

[11] Dipali Koshti, Supriya Kamoji, "Comparative study of Techniques used for Detection of Selfish Nodes in Mobile Ad hoc Networks", International Journal of Soft Computing and Engineering (IJSCE) ISSN: 2231-2307, Volume-1, Issue-4, September 2011.

[12] Khairul Azmi Abu Bakar and James Irvine Department of Electronic \& Electrical Engineering University of Strathclyde, “Contribution Time-based Selfish Nodes Detection scheme", ISBN: 978-1-902560-24-3 (c) 2010 PGNet.

[13] Martin Schütte, "Detecting Selfish and Malicious Nodes in MANETs", seminar: sicherheit in selbstorganisierenden netzen, hpi/universität potsdam, sommersemester 2006.

[14] S. Usha,member, IACSIT and S. Radha, "Multi Hop Acknowledgement Scheme based Selfish Node Detection in Mobile Ad hoc Network", international Journal of Computer and Electrical Engineering, Vol.3, No. 4, August 2011.

[15] Martin Schütte, "Detecting Selfish and Malicious Nodes in MANETs", seminar: sicherheit in selbstorganisierenden netzen, hpi/universität potsdam, sommersemester 2006.

[16] TAKAHIRO HARA, "Replica Allocation Methods in Ad Hoc Networks with Data Update", Mobile Networks and Applications 8, 343-354, 2003.

[17] Jae-Ho Choi, Kyu-Sun Shim, SangKeun Lee, and Kun-Lung Wu, "Handling Selfishness in Replica Allocation over a Mobile Ad Hoc Network", IEEE TRANSACTIONS ON MOBILE COMPUTING, VOL. 11, NO. 2, FEBRUARY 2012. 